\title{
Evaluation of the Thermo-Elastic Behavior of a High-alloyed Steel by Fourier Transformation based Lock-In-Thermography
}

\author{
by A. R. Urbanek*, J. Bär*
}

\begin{abstract}
* Institute of Materials Science, University of the Bundeswehr, Werner-Heisenberg-Weg 39, 85577 Neubiberg, Germany, ralf.urbanek@unibw.de,juergen.baer@unibw.de
\end{abstract}

\begin{abstract}
For the measurement of elastic stresses with Thermographic Stress Analysis a precise knowledge of the thermoelastic behavior also under consideration of plasticity and strain hardening is necessary. The influence of the coating and the specimen motion have been evaluated. The evaluation of the thermographic measurements was performed with a common sinus fit and a signal dissection with a discrete Fourier transformation (DFT). The distortion of the thermal response by plastic deformation is a known fact also the accompanying high harmonic parts in the DFT. The distortion of the thermal signal has been evaluated by using the Total Harmonic Distortion ratio.
\end{abstract}

\section{Introduction}

Lock-In-Thermography (LI-T) is a common technique for determining local stresses in cyclic-loaded components. This so-called Thermographic Stress Analysis (TSA) uses the cyclic change of the temperature, which results from the elastic stress by the thermoelastic effect given by the Thomson equation [1]:

$$
T(t)=-T_{m} \cdot K_{0} \cdot \sigma_{A} \cdot \sin \left(2 \pi f_{L} \cdot t\right) \quad \text { with } K_{0}=\frac{\alpha}{\rho \cdot c_{p}}
$$

The thermoelastic constant $K_{0}$ can be calculated from the density $\rho$, the specific heat capacity $c_{p}$ and the coefficient of thermal expansion $\alpha$. Due to the poor thermal emissivity of the metallic materials, the specimens have to be painted to enhance the emissivity of the surface. Unfortunately, because of the not ideal emissivity of the coating, the temperature amplitude measured by thermography is smaller than the theoretical values. When the thermal emissivity of the coating is unknown, a thermoelastic constant $\mathrm{K}_{\mathrm{E}}$ including the emissivity of the specimen surface can be determined by calibrating the system with defined stress amplitudes [2].

To reduce the noise in the measured temperature signal, the evaluation is performed using a DFT. Brémond [3] and Sakagami [4] have shown that in case of plastic deformation a second mode, coupled with the double loading frequency appears in the DFT-evaluation. This part is known as the so-called D-Mode and is assigned to dissipative energies. Urbanek and Bär [5] showed that in case of plastic deformation additionally amplitudes at higher harmonic frequencies appear, whose entities are currently unclear. The resulting complete DFT evaluation is given by equation (2):

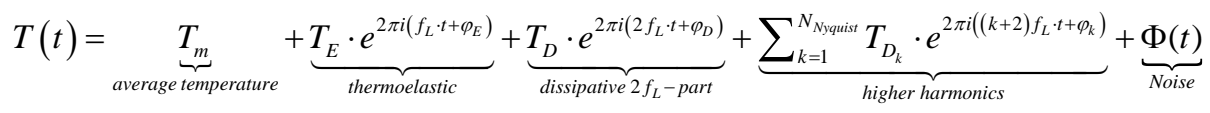

An other explanation for harmonic responses is given by Wong et al [6]. They stated that the thermoelastic constant is influenced by the mean stress and a temperature dependence of the Young's modulus (equation (3)).

$$
T(t)=-\frac{T_{m}}{\rho_{0} \cdot c_{p}}\left(\left(\alpha-\frac{1}{E^{2}} \frac{\partial E}{\partial T} \sigma_{m}\right) \sigma_{A} \cdot \sin \left(2 \pi f_{L} \cdot t\right)+\frac{1}{4 E^{2}} \frac{\partial E}{\partial T} \sigma_{A}^{2} \cdot \sin \left(2 \pi f_{L} \cdot t\right)\right)
$$

\section{Experimental details}

\subsection{Material properties}

In this work a high alloyed austenitic steel (X2CrNi 19-11, AISI 304 L) was investigated. The chemical composition of this alloy is shown in table 1 [7]. 
Table 1. Chemical composition of X2CrNi 19-11 (in weight percent).

\begin{tabular}{|c|c|c|c|c|c|c|c|}
\hline $\mathbf{C}$ & Si & Mn & $\mathbf{P}$ & $\mathbf{S}$ & $\mathbf{N}$ & $\mathbf{C r}$ & $\mathbf{N i}$ \\
\hline$\leq 0,03$ & $\leq 1,00$ & $\leq 2,00$ & $\leq 0,045$ & $\leq 0,015$ & $\leq 0,11$ & $18,00-20,00$ & $10,00-12,00$ \\
\hline
\end{tabular}

The physical parameters of the alloy are needed to determine the thermoelastic constant $\mathrm{K}_{0}$. The corresponding properties taken from the data sheet of the alloy [7] are given in table 2.

Table 2. Physical properties of X2CrNi 19-11 [7].

\begin{tabular}{|c|c|c|}
\hline $\begin{array}{c}\alpha \\
{[\mathbf{1} / \mathbf{K}]}\end{array}$ & $\begin{array}{c}\boldsymbol{\rho} \\
{\left[\mathbf{k g} / \mathbf{m}^{3}\right]}\end{array}$ & $\begin{array}{c}\mathbf{C}_{\mathbf{p}} \\
{[\mathbf{J} / \mathbf{k g} / \mathbf{K}]}\end{array}$ \\
\hline $16 \cdot 10^{-6}$ & 7900 & 500 \\
\hline
\end{tabular}

The mechanical properties were determined in tensile tests. The experiments have been performed with two different strain rates, $10 \mathrm{~mm} / \mathrm{min}$ and $100 \mathrm{~mm} / \mathrm{min}$, respectively. The yield strength $R_{p 0,2}$ was found to be $7 \%$ higher at 100 $\mathrm{mm} / \mathrm{min}$ and the strain to failure $A$ is about $10 \%$ lower. The ultimate tensile strength is not influenced by the strain rate. The steel shows a high strain to failure of about $44 \%$ at a strain rate of $10 \mathrm{~mm} / \mathrm{min}$. The mechanical properties are shown in table 3. Due to the constant loading frequency, the resulting mean strain rates in the fatigue tests depend from the maximum stress. They are about $28 \mathrm{~mm} / \mathrm{min}$ at $300 \mathrm{MPa}$ and about 50mm/min at 500MPa.

Table 3. Mechanical properties of X2CrNi 19-11.

\begin{tabular}{|c|c|c|c|}
\hline $\begin{array}{c}\text { Strain rate } \\
{[\mathrm{mm} / \mathrm{min}]}\end{array}$ & $\begin{array}{c}\mathbf{R}_{\mathbf{p 0}, \mathbf{2}} \\
{[\mathrm{MPa}]}\end{array}$ & $\begin{array}{c}\mathbf{R}_{\mathbf{m}} \\
{[\mathrm{MPa}]}\end{array}$ & $\begin{array}{c}\mathbf{A} \\
{[\%]}\end{array}$ \\
\hline 10 & 306 & 605 & 44 \\
\hline 100 & 325 & 605 & 40 \\
\hline
\end{tabular}

The fatigue experiments were carried out on flat specimens with a gauge length of $20 \mathrm{~mm}$ directly made from the sheet material. Figure 1 shows the technical drawing of the specimen. The gauge length has a width of $20 \mathrm{~mm}$, the specimen thickness is $3 \mathrm{~mm}$. To enhance the emissivity, the surface in the gauge length was grinded and coated with a black paint or graphite spray, respectively. The black paint was applied with a mean thickness of $20 \mu \mathrm{m}$, the graphite coating with a mean thickness of about $5 \mu \mathrm{m}[8]$.

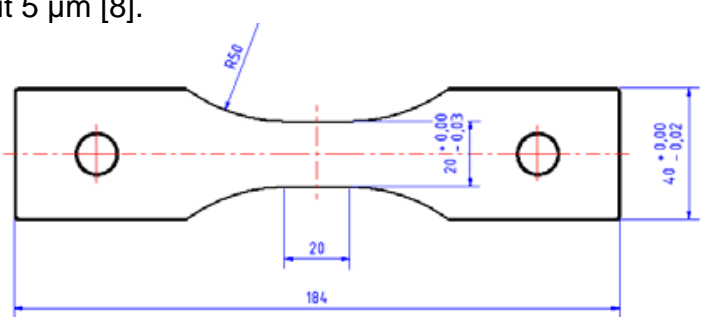

Fig. 1: Technical drawing of the specimen.

The fatigue experiments were carried out with a servo-hydraulic testing machine with a load ratio of $R=0$ and a loading frequency of $5 \mathrm{~Hz}$. The loading level was increased in steps of $25 \mathrm{MPa}$ every 1,200 cycles starting from an initial maximum stress of $25 \mathrm{MPa}$.

\subsection{Lock-In-Thermography}

The thermographic measurements were performed with an InfraTec ImageIR $8300 \mathrm{hp}$ camera. The general properties of the camera are given in table 4.

Table 4. Camera properties of ImageIR® $8300 \mathrm{hp}$

\begin{tabular}{|l|l|}
\hline Detector material & InSb \\
\hline Number of pixel & $640 \times 512$ (full frame) \\
\hline Spectral response & $2,0-5,7 \mu \mathrm{m}$ \\
\hline Focal length & $50 \mathrm{~mm}$ with $11 \mathrm{~mm}$ distance ring \\
\hline NETD & $<20 \mathrm{mK}$ at $30^{\circ} \mathrm{C}$ \\
\hline
\end{tabular}


To enhance the recording frequency of the camera the measurements were performed with a quarter frame of $160 \times 125$ pixel and an integration time of $460 \mu \mathrm{s}$. To avoid a multiple of the loading frequency the recording frequency of the camera was set to $997 \mathrm{~Hz}$. A single measurement consists of 9970 frames and lasts $10 \mathrm{~s}$. In order to record the temperature in a thermal equilibrium the last 300 cycles of each load step were recorded. For this, the first thermographic measurement was started manually and the following measurements were started automatically in intervals of 4 minutes. To enable a synchronisation of the thermographic measurements with the mechanical loading, the force signal of the testing rig was transferred as an analog signal to the camera and stored with each recorded frame.

\subsection{Common analysis of the thermoelastic effect}

The common analysis of the thermoelastic effect bases on the adopted version of the Thompson equation (Eq. (1).) The average temperature of all pixel in a single frame and the corresponding force value from each frame are plotted as a function of the time. The temperature curve is fitted using a common sinusoidal function given by equation (4):

$$
T(t)=T_{m}+T_{E} \cdot \sin \left(2 \pi f_{L} \cdot\left(t+t_{T}\right)\right)
$$

This adjustment results in values for the mean temperature $T_{m}$, the temperature amplitude $T_{E}$ and the time shift $t_{T}$ between the temperature and the force signal. According to equation (1) the force and the temperature should be in counter shift. In case of a loading frequency of $5 \mathrm{~Hz}$ the resulting time shift should be $t_{\mathrm{T}}=100 \mathrm{~ms}$.

\subsection{DFT analysis of the thermoelastic effect}

The DFT analysis of the temperature signal results in a dissection of the measured signal for each pixel in a sum of sinusiodal waves according to equation (2). In contrast to the common evaluation with a simple sinusoidal fit, beside the thermoelastic response, values for the amplitude and the phase shift for the dissipative $2 \mathrm{f}_{\mathrm{L}}$-part and the higher harmonic frequencies up to the Nyquist frequency are achieved. The Nyquist frequency is determined by the recording frequency (sampling rate) of the camera and is calculated to about $498 \mathrm{~Hz}$ [9]. Due to the DFT evaluation, the noise of the temperature estimation is minimized. The noise level is determined to $0.6 \cdot \mathrm{mK}$ by a conservative estimation.

Figure 2 shows the amplitude spectrum of a complete DFT according to equation (2). The peak at the frequency $\mathrm{f}=0 \mathrm{~Hz}$ represents the mean temperature $T_{\mathrm{m}}$. In case of pure elastic loading only one additional peak at the loading frequency of $f=5 \mathrm{~Hz}$ appears (red circles in figure 2). The small peak at $30 \mathrm{~Hz}$ is probably caused by the vibration of the testing rig.

When the specimen is loaded above the elastic limit, peaks at each multiple of the loading frequency appear (black line in figure 2). This clearly indicates that the higher harmonic frequencies are coupled with plastic deformation and it is not sufficient to describe the dissipated energies only with the $2 \mathrm{fL}_{\mathrm{L}}$-part.

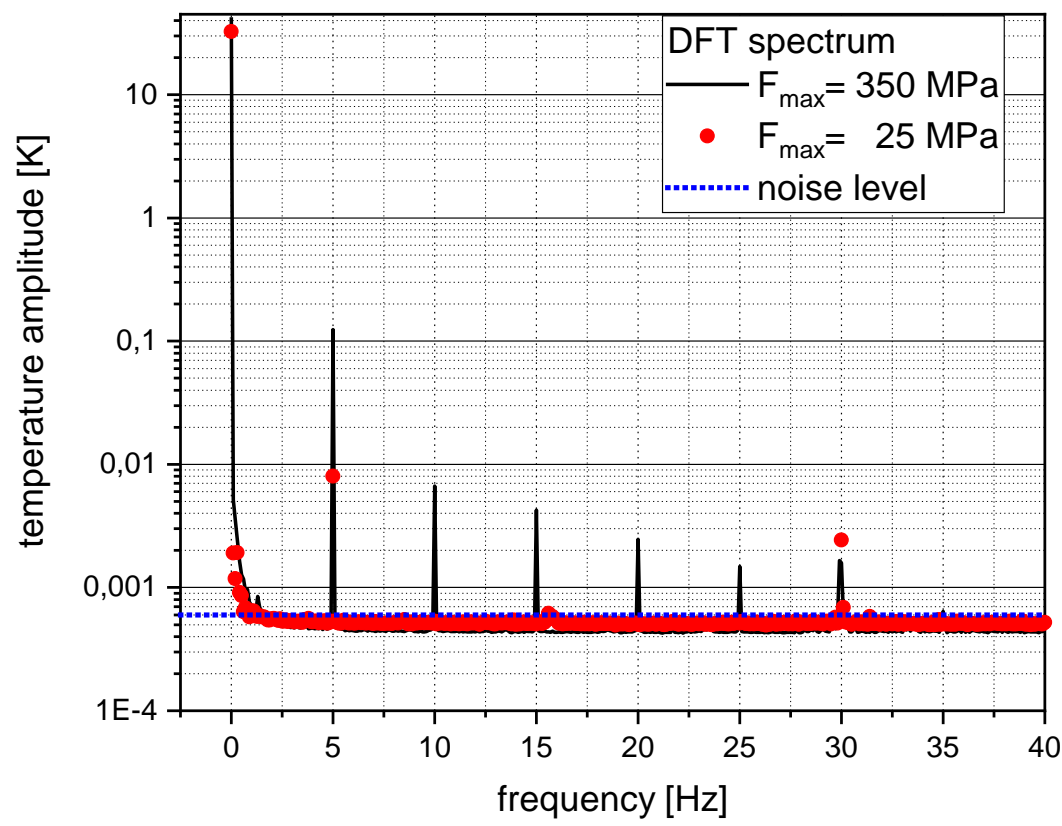

Fig. 2: Complete temperature DFT dissection.

When the rigid body motion of the specimen is not negligible, the evaluation should be combined with a motion compensation technique previously used by Urbanek and Bär [2,5]. This compensation technique requires sharp 
gradients, for example the border of the painted area. The usability of the motion compensation (MC) was not the primary task of this investigation, therefore no special gradients were implemented. Nevertheless, MC could be adopted to spontaneous occurring patters like small cracks or crazes in the coating.

\section{Experimental results}

\subsection{E-Amplitude in Common and Lock-In evaluation}

Figure 3 shows the results of the common and DFT-analysis for the specimens with coated with black paint (a) and graphite (b), respectively. The relative temperature amplitude $T_{E} / T_{m}$ shows a linear dependence of the applied stress within the elastic regime up to a maximum stress of $300 \mathrm{MPa}$. This correlation is supported by equation (1). A prestraining of the specimen prior to the experiment has no influence on the thermoelastic effect.
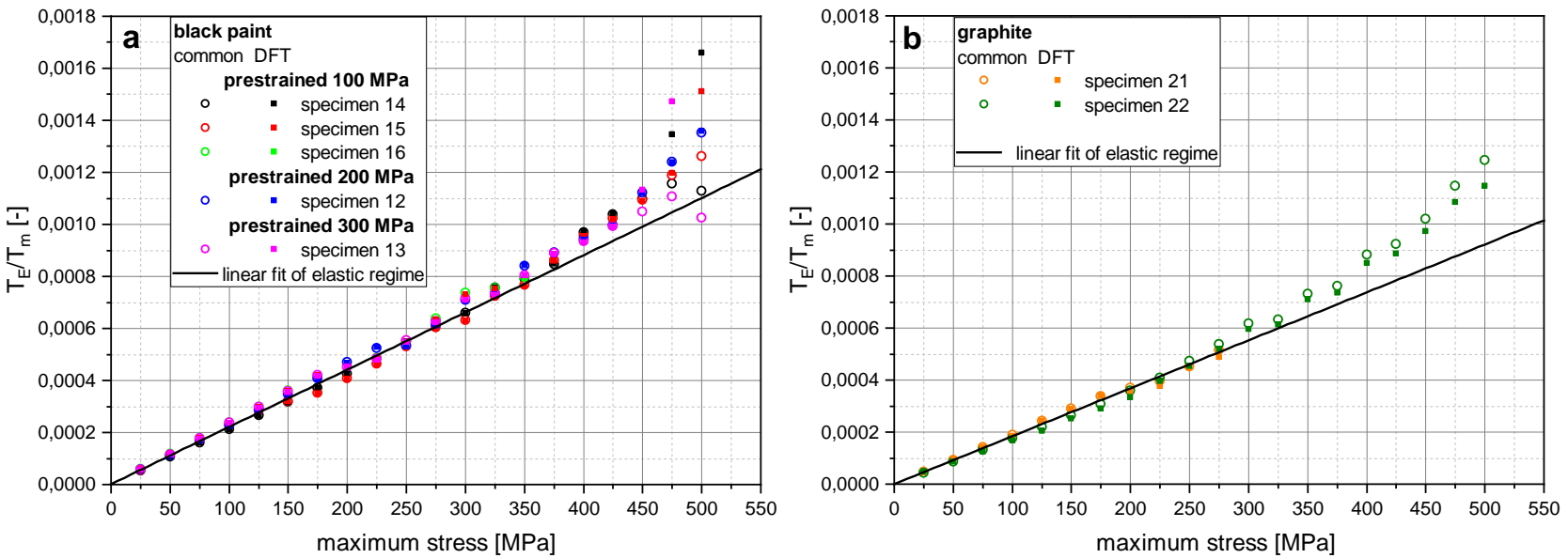

Fig. 3: Relative thermoelastic temperature for both analysis with black paint a) and graphite.

The maximum stress in the fatigue experiments shown in figure 3 is the double of the stress amplitude. Therefore, the thermoelastic constant $\mathrm{K}_{\varepsilon}$ is the proportional factor which can be easily determined by a linear regression of the elastic regime in the section $[0 \mathrm{MPa} / 275 \mathrm{MPa}$ ]. The results of this regression with coefficient of determination (COD) and resulting $\mathrm{K}_{\varepsilon}$ are shown in table 5 . The theoretic value $\mathrm{K}_{0}$ determined with equation (1) and the physical properties given in table 2 is shown for comparison.

Table 5. Results of thermoelastic constant.

\begin{tabular}{|c|c|c|c|c|}
\hline & Analysis & $\begin{array}{c}\text { Slope } \\
{[1 / \mathrm{MPa}]}\end{array}$ & COD & $\begin{array}{c}\mathrm{K}_{\varepsilon}\left(\mathrm{K}_{0}\right) \\
{[1 / \mathrm{MPa}]}\end{array}$ \\
\hline black paint & DFT & $2,193 \cdot 10^{-6}$ & 0,998 & $4,385 \cdot 10^{-6}$ \\
\hline & Common & $2,218 \cdot 10^{-6}$ & 0,998 & $4,437 \cdot 10^{-6}$ \\
\hline graphite & DFT & $1,847 \cdot 10^{-6}$ & 0,998 & $3,694 \cdot 10^{-6}$ \\
\hline & Common & $1,842 \cdot 10^{-6}$ & 0,998 & $3,684 \cdot 10^{-6}$ \\
\hline theoretic Ko & & & & $4,051 \cdot 10^{-6}$ \\
\hline
\end{tabular}

The common and the DFT analysis show the expected linear behavior in the presumed elastic regime and lead to the same good coefficient of determination. The measured value for $\mathrm{K}_{\varepsilon}$ should be smaller than the theoretical value $\mathrm{K}_{0}$, due to the reduction of the signal caused by the not ideal thermal emissivity of the paint. In case of the black paint, the measured value is higher than the theoretical one. This discrepancy is caused by uncertainties of the values taken from the data sheet (table 2) for the determination of $\mathrm{K}_{0}$ with equation (1). These values should be determined for the special batch of material used in this investigation. This determination is expensive and time-consuming, in addition the emissivity of the coating is still unknown. A more promising method is the experimental determination of a thermoelastic constant $\mathrm{K}_{\varepsilon}$, which includes the emissivity of the coating. For this, fatigue tests with a stepwise increase of the applied load as shown in figure 3 seem to be the appropriate method.

Above a maximum stress of $300 \mathrm{MPa}$ the relative temperature amplitude starts to drift from the linear slope to higher values. An increase is not supported by equation (2) given by Wong et al. [6]. Therefore, a mean stress dependence for the investigated material can be denied. 
With increasing load level, the elongation of the metal exceeds the maximum elongation of the black coating. Figure 4 shows exemplarily the damages of the black paint (a) and the graphite coating (b) after a complete fatigue experiment, i.e. after loading with a maximum stress of $500 \mathrm{MPa}$. The cracks in the coating form perpendicular to the loading direction. The transverse contraction can stimulate a separation of coating and metallic surface. Therefore, larger cracks in the black paint are accompanied with areas of ablation of the coating. In the graphite coating the graphite particles stick independently on the surface and form only a slight coherent painting. In the graphite coating the crazing pattern has a finer structure compared to the black paint and ablation of the coating is less pronounced.
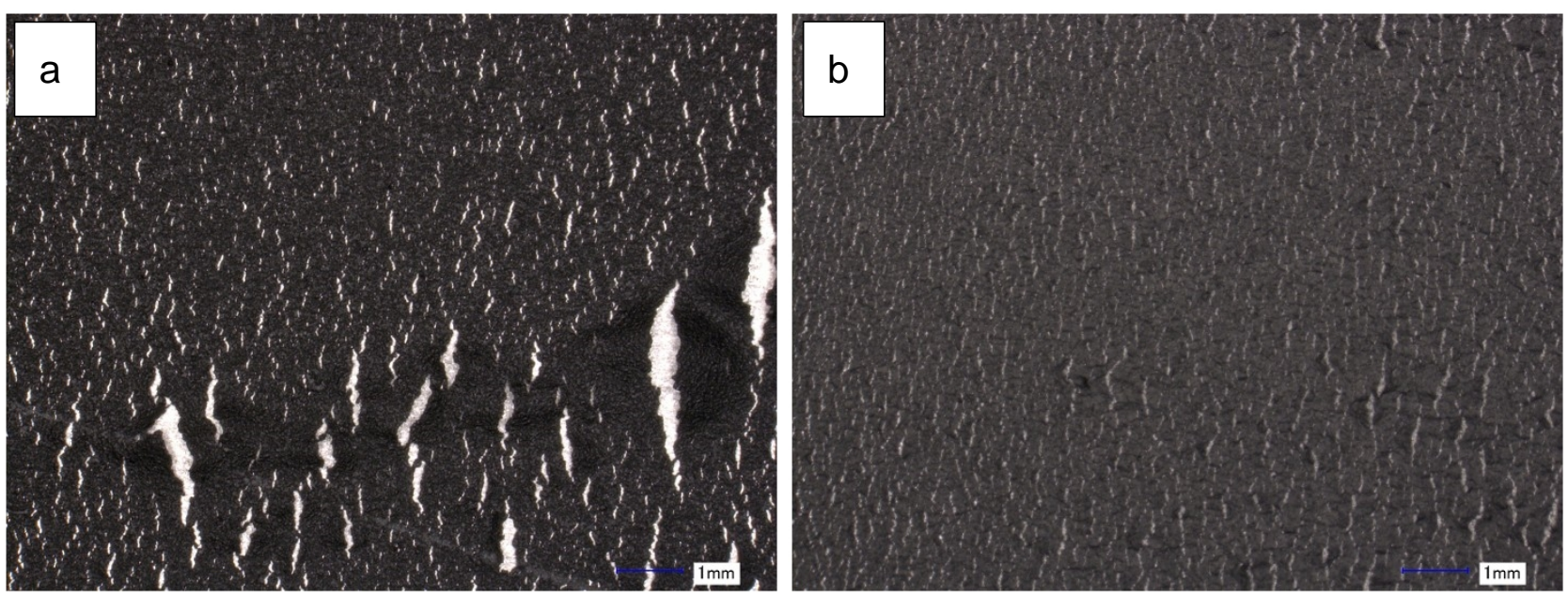

Fig. 4: Cracks and crazes in black paint (a) and graphite coating (b).

The stress when cracks are initiated and the number and size of cracks and crazes differ from specimen to specimen. Generally, at a load level of $350 \mathrm{MPa}$ in the black paint small crazes are formed and at $425 \mathrm{MPa}$ huge cracks in coating become visible. The graphite coating does not form a coherent coating like the black paint, therefore only the distance between the individual particles increase. Thereby, the crazing starts at lower stress levels but even at higher loads no large cracks are visible. In both coatings the cracks and crazes are not equally distributed on the surface. By avoiding areas with large cracks, the deviation from the linear slope can be reduced.

Beside the damage in the coating, the reduction of the cross section due to plastic deformation influences the results. The resulting stress in the experiments is calculated by using the starting cross section in the gauge length. Due to the high elongation of the steel the reduction of the specimen cross section is not negligible. The increase of the maximum stress causes a reduction of the cross section and therefore the true stress is increased.

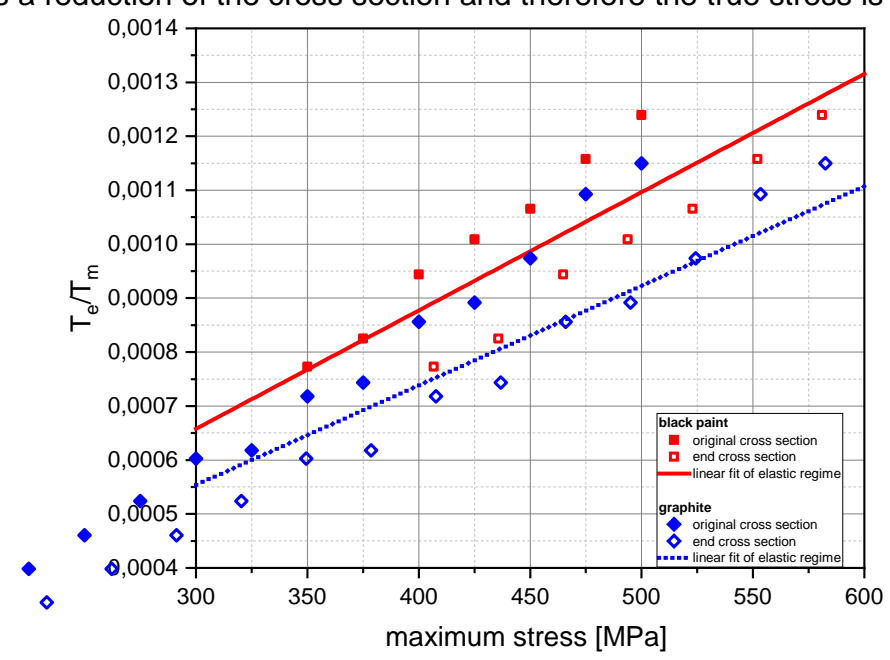

Fig. 5: Influence of reduced specimen cross section.

Figure 5 shows the results for black paint and the graphite coating by avoiding areas with large cracks. Filled symbols show the stress values calculated with the original cross section, whereas the open symbols represent the stress calculated with the final cross section. The solid lines indicate the thermoelastic response calculated with equation (1) and the experimental $\mathrm{K}_{\varepsilon}$-values (table 5). For higher stresses the values calculated with the final cross section are closer to the 
estimated thermoelastic slope. This result clearly indicates that the reduction of cross section has to be recognized to get reliable results in TSA measurements in the elastic-plastic regime.

From the TSA investigations a few important conclusions can be stated:

- $\quad$ The thermoelastic constant is independent from the mean stress.

- The thermoelastic constant is not influenced by the strain hardening or plastic deformation.

- The analysis via DFT and the common evaluation produce the same results.

- The debonding behavior and the ductility of the coating is a crucial issue for TSA measurements at high deformations.

\subsection{E-Phase in common and Lock-In evaluation}

The thermoelastic effect was firstly described by Thompson for the temperature change during the compression and decompression of gaseous materials [1]. By reducing the volume under adiabatic conditions the specific average energy hence the temperature increases. In thermal equilibrium under cyclic loading the temperature signal should be in counter shift to the loading signal. The phase shift in the fatigue tests with a frequency of $5 \mathrm{~Hz}$ should be $100 \mathrm{~ms}$ in perfect counter shift. The experimental phase shift was calculated according to equation (5):

$$
\Delta t_{d}=t_{T}-t_{F}-\frac{1}{2 f_{L}}=t_{T}-t_{F}-100 m s
$$

In figure 6 the phase shift as a function of the maximum stress for specimens coated with black paint is shown. A phase shift of zero represents a perfect accordance to the basic equation (1), thus counter shift behaviour. Up to a stress of $425 \mathrm{MPa}$, although above $300 \mathrm{MPa}$ plastic deformation takes place, the phase shift for all investigated specimens is nearly constant and close to zero within a scatter band of $\pm 1 \mathrm{~ms}$. The evaluation with a common fit shows the same run as the DFT but is shifted about 0,6 ms to slightly higher values. The scatter band is in the same dimension like the integration time of the camera with $0,46 \mathrm{~ms}$ and the sampling interval with 1,003 $\mathrm{ms}$.

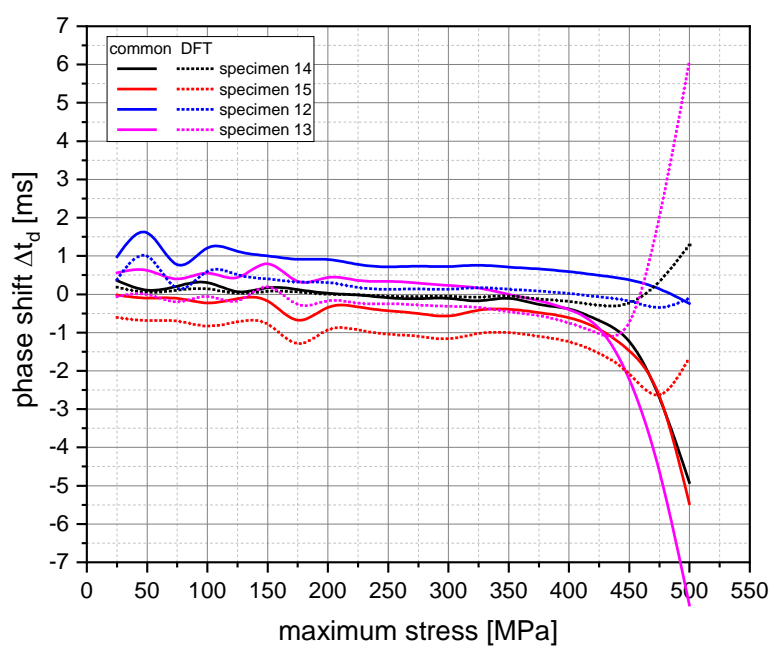

Fig. 6: Phase shift as a function of applied stress for the different evaluation methods.

At maximum stresses above $425 \mathrm{MPa}$ the phase shift determined with the common method decreases rapidly to negative values of about $7 \mathrm{~ms}$. The results of DFT method show a distinct change without a clear trend. The changes in the phase shift can be contributed to the occurrence of crazes and cracks. This is supported by the results obtained on specimen 12, where only minor crazes were found and, consequently, the phase shift remains constant close to zero. Depending on the evaluation method the generation of crazes and cracks results in different phase shift values. These differences are due to the differences in the evaluation processes. In the common method in a first step the average temperature of every thermographic image is calculated and afterwards a sinusoidal fit with this average values according to equation (2) is performed. In the DFT method first the DFT-Analysis for each pixel is carried out and then the average value of the time shift of all pixels is calculated. Obviously, these differences in the evaluation processes are responsible for the differences in the results. Nevertheless, the damage of the coating influences the phase results.

In figure 7 the influence of the damage in the black paint and graphite coating for both analysis methods is shown. For the black paint values for an evaluation in a region with small cracks is compared with a region with large cracks and for the graphite coating a region with a low crack density is compared with a region with a high crack density. Before discussing the results, the general damage process of black paint and graphite has to be outlined. The black paint forms a coherent surface which can follow the strain of specimen without any damage up to $350 \mathrm{MPa}$. With increasing maximum 
stress small crazes were formed in the coating. At a load level of about $425 \mathrm{MPa}$ in some specimen large cracks become visible. The graphite coating shows a damage behavior without generating large cracks. The crazes formed in the graphite coating are small and show an equal distribution, but the development of crazes starts already at the level of the yield strength $\mathrm{R}_{\mathrm{p} 0,2}$ of about $300 \mathrm{MPa}$.

The developing of crazes and cracks result in a phase shift in both analysis methods. The change of the phase shift starts at the previous mentioned stress levels. The damage of the coating itself is not the crucial topic for the phase shift, because the cracks and crazes only influence the emissivity. Unless the emissivity is on a sufficient level, no phase shift should occur, only the amplitude values are influenced.
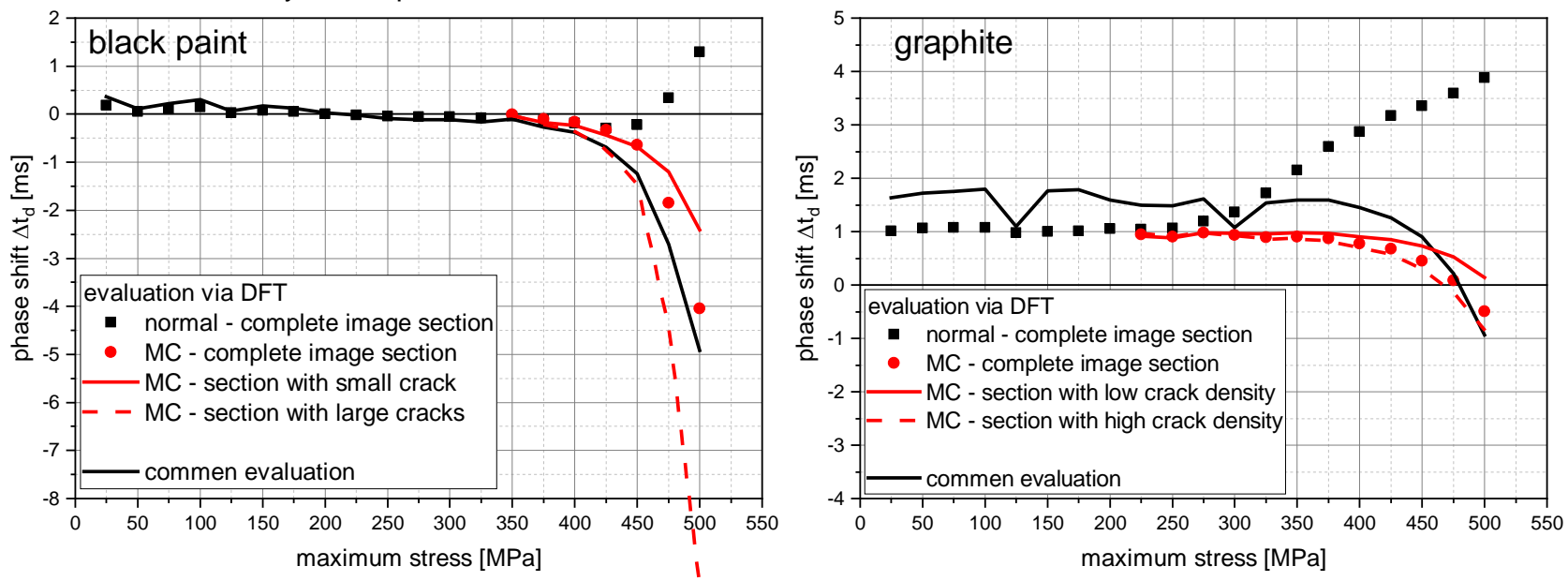

Fig. 7: Influence of crazes and cracks in the coating and rigid body motion on the phase.

The more crucial point seems to be the rigid body motion of the specimen. This effect can be reduced by using a $\mathrm{MC}$ technique. For the application of the motion compensation recognisable patterns are necessary. Therefore, normally the border of the painted region is used. The usage of MC was not the primary object of this investigation, therefore these patterns had not been applied. To enable the usage of the MC, cracks in the coating were used as auxiliary patterns. This requires a minimum level of damage; thus the MC could not be applied at low maximum stresses. The red lines in figure 7 show the results corrected with MC.

The subjective impression, that damage in the graphite coating occurs at lower stresses compared to black paint, is supported by the stress level of the first applicability of the MC. Due to the application of the MC, the results of the DFT evaluation were shifted towards the results of the common evaluation. This effect is more pronounced for the black paint compared to the graphite coating. This supports the impression that the DFT is more sensitive to motion than the common evaluation. To evaluate the influence of the cracks and crazes, different regions on the specimen were investigated. Therefore, a region with large cracks (high crack density) and one with smaller cracks (low crack density) was analysed. In both regions the general shape of the phase shift trajectory is not changed. The changes of the phase shift are directly connected with the size and the number of cracks. The evaluation of the graphite coating shows, that even a high number of small crazes in combination with the rigid body motion of the specimen causes significant changes in the phase shift.

Considering the specimen motion in combination with the damage of the coating, the phase shift between the force and of the temperature signal of the thermoelastic effect can be presumed to be constant and in counter shift. A change of the phase shift due to plastic deformation cannot be observed. In consequence, the investigations of the cyclic plastic zone ahead of a crack tip based on the phase shift between the force and the thermoelastic response undertaken by Tomlinson and Patterson [10] as well as Bär and Seifert [11] may be contributed to cracks in the coating due to high plastic deformation.

\subsection{Analysis of higher harmonics in Lock-In evaluation}

In Lock-In experiments the measured effect is modulated with a frequency. In thermoelastic induced Lock-In thermography this frequency is the loading frequency. Assuming linear elastic material behaviour, the thermal response should only appear with this frequency. Higher harmonics (multiple of the loading frequency) in a DFT decomposition are needed to describe a periodic non sinusoidal signal, for instance a rectangular or triangle shaped signal. In our case the appearance of higher harmonics indicates a deformation of the presumed sinusoidal signal.

Figure 8a clearly shows appearance of the higher harmonics at higher maximum stress. The resolution limit is about $1 \mathrm{mK}$. This limit is nearly twice the noise level. This increased resolution limit is owed to a limited scale in the file format used for the analysis. At lower stress levels the values of the higher harmonics are in the dimension of the resolution limit. In figure $8 \mathrm{~b}$ the general trend of all considered higher harmonics is shown. All higher harmonies show a similar linear rising slope in linear-log diagram.

Sakagami [4] showed that the mentioned deviation could mainly be described by the first harmonic (2f-part) in experiments with alternating loads, forcing plastic deformation in tension and compression. He defined the $2 f-p a r t$ as $D-$ 
Mode and used it as an indicator for the identification of the plastic zone in front of a crack. In experiments with unsymmetrical loading this simple approach is not sufficient. A more suitable approach might be the description of the deviation by using the Total Harmonic Distortion (THD). The THD is a dimensionless ratio which is computed from the amplitudes of the higher harmonics and the amplitude of the loading frequency [12].
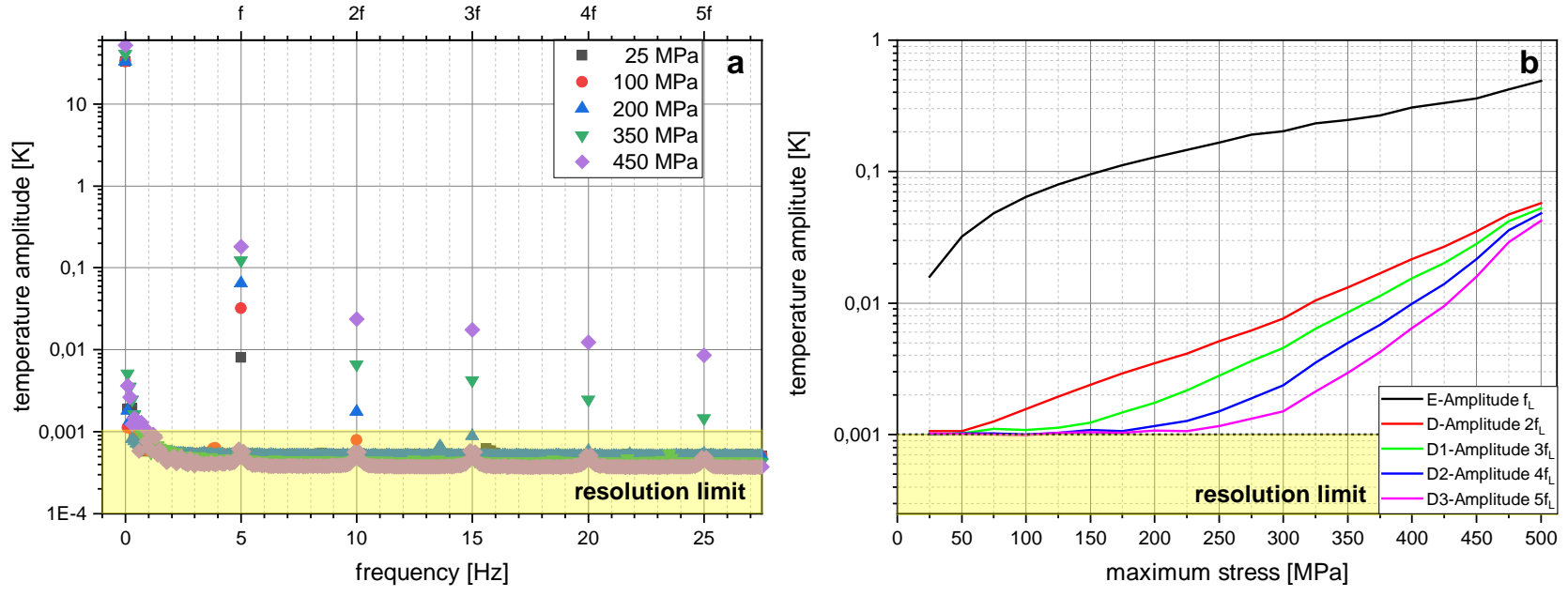

Fig. 8: DFT dissection of different load levels.

Above a maximum stress of $275 \mathrm{MPa}$ all amplitudes are significantly above the resolution limit (figure $8 b$ ). The calculation of the THD was limited to the four lowest higher harmonics. The corresponding approach for the $\mathrm{THD}_{4}$ is given in equation (6). The THD calculation with the square of the amplitude values is suitable for energies.

$$
T H D_{4}=\frac{T_{D}{ }^{2}+T_{D_{1}}{ }^{2}+T_{D_{2}}{ }^{2}+T_{D_{3}}{ }^{2}}{T_{E}{ }^{2}}
$$

The results of the $\mathrm{THD}_{4}$ for the black paint are shown in figure 9. All specimens show generally the same run in the THD curve. No influence of the prestraining can be observed. The $\mathrm{THD}_{4}$ values decrease for rising maximum stress up to about $125 \mathrm{MPa}$ and then they remain nearly constant up to a maximum stress of about $250 \mathrm{MPa}$. The increase after the constant level depends on the development of crazes and cracks.

The decrease of the THD values can be contributed to the decrease of the resolution limit and the small values of $T_{E}$ (thermoelastic effect). This determines a resolution limit for the THD depending of the resolution limit or noise level and the temperature amplitude of the thermoelastic effect.

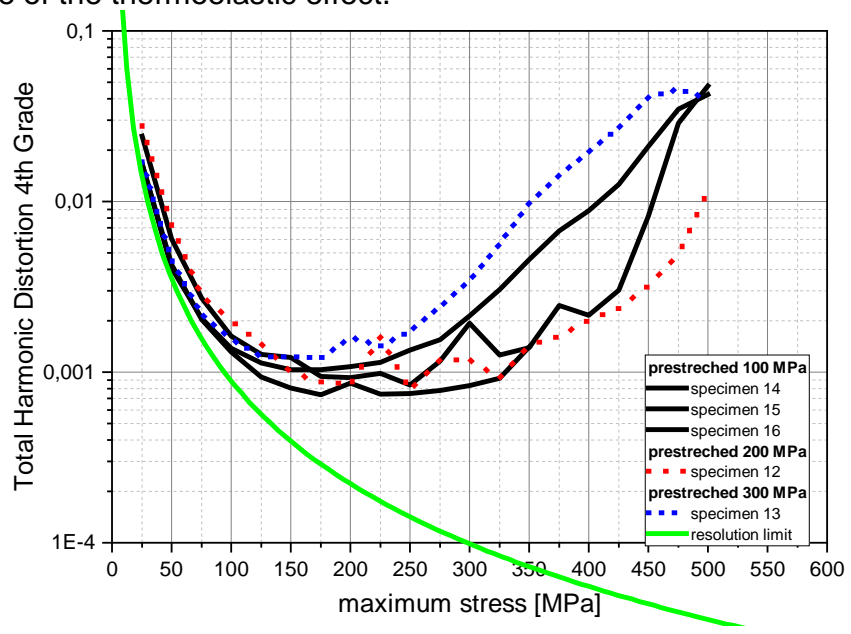

Fig. 9: Total harmonic distortion of specimens with black paint

The resolution limit for the THD can be calculated with equation (7) considering the number of accounted harmonics $\mathrm{n}$, the resolution limit of temperature amplitudes Tresolution limit and the temperature amplitude of the presumed thermoelastic effect $\mathrm{T}_{\mathrm{E}}$ : 


$$
T H D_{\text {limit }}=\frac{n \cdot\left(T_{\text {resolution limit }}\right)^{2}}{T_{E}^{2}}=\frac{4 \cdot(0,001[K])^{2}}{T_{E}^{2}}
$$

The THDlimit precisely characterises the decrease of the THD for low maximum stresses. The constant values of THD at stresses around the yield strength and the following rise indicates the influence of plastic deformation. An influence of specimen motion due to cracks and crazes in the coating, which is described in chapter 3.2, may also explain the increasing values.

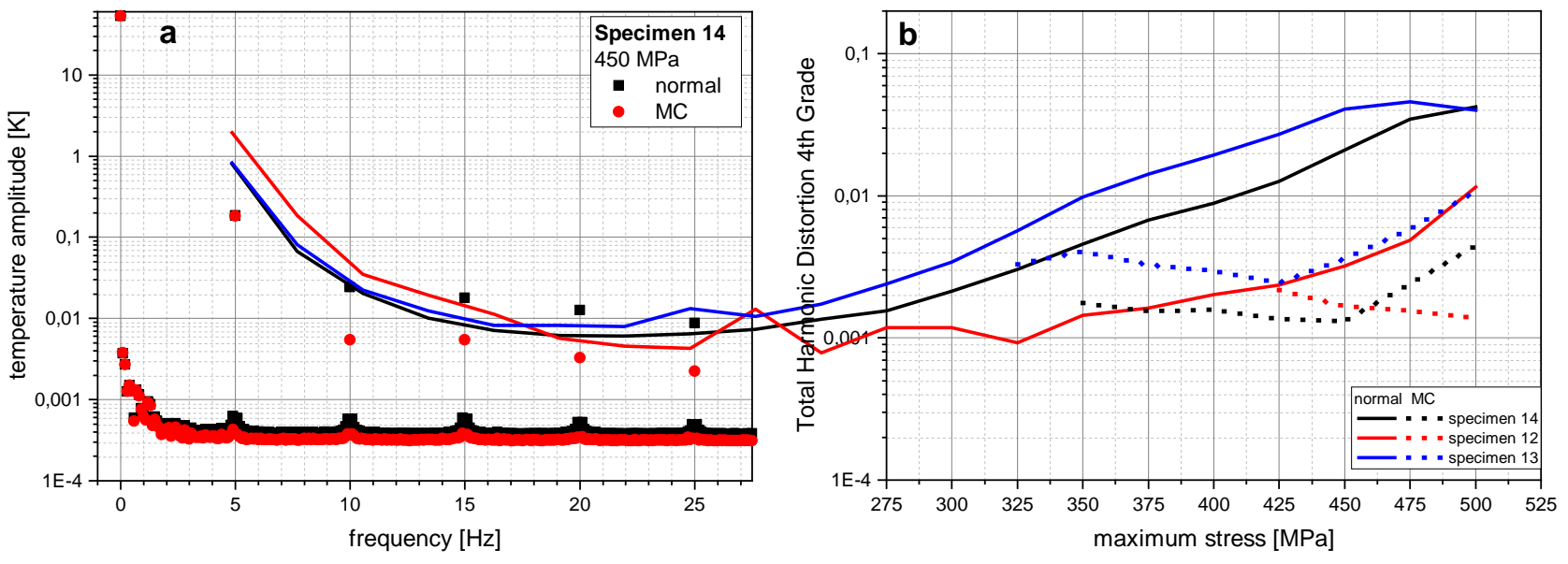

Fig. 10: Influence of motion on DFT total harmonic distortion.

To verify if the rise of THD is driven by rigid body motion, the results of the DFT with and without MC were compared. Figure 10a shows the influence of $\mathrm{MC}$ on the amplitude values. The figure shows the result of specimen 14 at a maximum stress of $450 \mathrm{MPa}$. The thermoelastic amplitude $\mathrm{T}_{\mathrm{E}}$ represented by the peak at $5 \mathrm{~Hz}$ is not affected by the MC. The level of the higher harmonics is reduced by about $50 \%$. This influences significantly the THD values.

This influence of the MC on the THD is shown in figure 10b for three different specimens coated with black paint. The increase of the THD for higher stresses is significantly reduced to the level of constancy starting at a stress level of $125 \mathrm{MPa}$, only a slight increase still remains. This remaining increase accords with the deviation of the E-Phase which indicates a non-negligible damage of the coating. The influence of the damage of the coating can only be reduced, but not completely compensated. The specimens with graphite coating show the same behavior. The decrease parallel to the resolution limit of the THD is followed by a level of constancy which remains up to high stresses, when the rigid body motion is compensated. The nominal level of constant THD is one dimension higher compared to the level obtained at specimens covered with black paint. This refers probably to the more incoherent structure of graphite already described in chapter 3.1. The following increase of the THD can be handled by MC as in the case of the black paint leading to a THD at the level of constancy.

The constancy of THD implies a constant distortion of the temperature signal. This constant distortion can be contributed to plastic deformation or to the uncompensated rigid body motion or the unconsidered strain. A measurement of the plastic deformation via THD without considering motion and the equality of the coating is impossible.

\section{Summary and consequences for evaluation of plastic deformation}

The investigated high-alloyed steel shows a high elongation to failure and a distinct strain hardening behavior. The measured thermal response was investigated by two different analysis methods. The investigation was carried out at three major issues: the relative amplitude of the thermoelastic effect $\left(T_{E} / T_{m}\right)$, the phase shift of the thermoelastic effect $\left(\Delta t_{d}\right)$ and the measurement of the signal distortion with THD based on the results of the DFT decomposition.

The results for both analysis methods lead to the same results. The determined thermoelastic constant according to Thompsons model is independent of the mean stress and the strain hardening of the material. The evaluation shows clearly the necessity of a coating that forms a coherent surface and shows elastic behaviour in the same order of the investigated material.

The results of the phase shift show a very good accordance for both methods. The phase shift between the thermoelastic effect and the applied force shows the predicted counter shift. The homogeneity of the coating as well as crazes and cracks in the coating influence the measured phase shift. The common method is less sensitive to small damages. Deviations caused by the inhomogeneity of the coating and crazes can be attenuated by eliminating the rigid body motion of the specimen. Actually large cracks can be treated this way, gaining a limited improvement of the results. This sensitivity to damage raise the questions: "What kind of entity is the phase shift in front of a crack described by Tomlinson [10] and Bär [11]? Is this a real material effect caused by large strains or just a surface effect caused by a 
damaged coating?" Unfortunately, in both publications no detailed examination of the coating in the area of the crack tip was carried out.

The distortion of the thermal response was evaluated by calculating the Total Harmonic Distortion. The THD is highly influenced by rigid body motion and the signal-to-noise ratio of the thermal response caused by the alternate loading. Evaluations, considering the specimen movement by using a motion compensation technique, show that THD hence distortion of the thermal response is constant in the elastic and the elastic plastic regime. The reason for the constancy can be the existence of a minor specimen movement, due to the limits of the used compensation technique or the unconsidered strain of the specimen. Nevertheless, the constant deviation can also be caused by plastic deformation despite the thermal equilibrium is reached. Both potential effects could not be separated in this experiment, but it will be task of future investigations. The constancy of the of THD shows that the deformation of the pure sinus is independent from the level of the thermoelastic response. The THD does not account the phase information of the higher harmonics, regardless the area normed by the thermoelastic effect under the curve remains constant.

\section{REFERENCES}

[1] Thomson, W. On the dynamical theory of heat. In: Transaction of the Royal Society of Edinburgh, 20, 1853, pp. 261-288

[2] Urbanek, R.; Bär J. Lock-In Thermographic Stress Analysis of notched and unnotched specimen under alternating loads, In: Procedia Structural Integrity 5, 2017, pp 785-792:

[3] Brémond, P., New developments in Thermo Elastic Stress Analysis by Infrared Thermography. In: IV Conferencia Panamericana de END 2007.

[4] Sakagami, T.; Kubo, S.; Tamura, E.; Nishimura, T.. Identification of plastic-zone based on double frequency lock-in thermographic temperature measurement, In: ICF11 Italy, 2005

[5] Urbanek, R.; Bär J. Influence of motion compensation on lock-In thermographic investigations of fatigue crack propagation. In: Engineering Fracture Mechanics, 2017. http://doi.org/10.1016/j.engfracmech.2017.03.043

[6] Wong, A. K.; Sparrow, J. G.; Dunn, S. A. 1988. On the revised theory of the thermoelastic effect. Journal of Physics and Chemistry of Solids 49, 395-400. DOI: 10.1016/0022-3697(88)90099-6

[7] ThyssenKrupp Materials Europe, Werkstoffdatenblatt TK. 1.4306, 03/2006

[8] Robinson, A. F.; Dulieu-Barton, J. M.; Quinn, S.; Burguete, R. L. 2010, Paint coating characterization for thermoelastic stress analysis of metallic materials. Measurement Science and Technology 21, DOI: 10.1088/0957-0233/21/8/085502

[9] Breitenstein, W.;Warta, W. Langenkamp, M. Lock-In Thermography -Basics and Use for Evaluating Electronic Devices And Materials. Springer Series in Advanced Microelectronics 10, 2010

[10] Tomlinson. R. A, Patterson E.A., Examination of crack tip plasticity using Thermoealstic Stress Analysis In. Proulx - Thermomechanics and Infra-Red Imaging 2011, P, 123-129, http://doi.org/10.1007/978-1-4614-02077 16

[11] Bär J., Seifert S., Investigation of Energy Dissipation and Plastic Zone Size during Fatigue Crack Propagation in a High-Alloyed Steel, In: Procedia Materials Science 3. 2014. p.408-413 http://doi.org/10.1016/j.mspro.2014.06.068

[12] Shmilovitz, D.; On the Definition of Total Harmonic Distortion and Its Effect on Measurement Interpretation, In: IEEE Transactions on Power Delivery, VOL. 20, NO. 1, 01/2005 